\title{
Supply and Demand Aware Synthetic Data Generation for On-demand Traffic with Real-world Characteristics
}

\author{
Reem Y. Ali \\ Computer Science \& Engineering \\ Dept., University of Minnesota \\ Minneapolis, MN \\ alireem@cs.umn.edu
}

\author{
Yan Li \\ Computer Science \& Engineering \\ Dept., University of Minnesota \\ Minneapolis, MN \\ lixx4266@umn.edu
}

\author{
Shashi Shekhar \\ Computer Science \& Engineering \\ Dept., University of Minnesota \\ Minneapolis, MN \\ shekhar@cs.umn.edu
}

\author{
Shounak Athavale \\ Ford Motor Company \\ Palo Alto, CA \\ sathaval@ford.com
}

\author{
Eric Marsman \\ Ford Motor Company \\ Dearborn, MI \\ emarsman@ford.com
}

\begin{abstract}
The on-demand economy has attracted significant attention in recent years, with a rapid growth in on-demand services ranging from ride-hailing to package delivery and grocery pickup. However, real-world spatio-temporal data that can be used for evaluating research on on-demand brokers design and supply-demand regulation are either not publicly available or are very limited in their spatial coverage. Research efforts in generating synthetic spatio-temporal datasets such as traffic generators have only focused on one side of the business model, particularly the demand side, and thus are not convenient for studying market variations such as the problem of supply-demand imbalance. In addition, many of these generators do not accurately reflect real-world data characteristics. In this paper, we propose a supply and demand aware framework for generating synthetic datasets for the purpose of designing on-demand spatial service brokers, while also capturing real-world data characteristics by leveraging multiple publicly available data sources. We also present an evaluation of the quality and performance of our proposed framework.
\end{abstract}

\section{CCS CONCEPTS}

- Information systems $\rightarrow$ Location based services; Geographic information systems;

\section{KEYWORDS}

supply and demand aware, synthetic data generation, on-demand services, on-demand brokers, spatial service broker, synthetic data with real-world characteristics

Permission to make digital or hard copies of all or part of this work for personal or classroom use is granted without fee provided that copies are not made or distributed for profit or commercial advantage and that copies bear this notice and the full citation on the first page. Copyrights for components of this work owned by others than ACM must be honored. Abstracting with credit is permitted. To copy otherwise, or republish, to post on servers or to redistribute to lists, requires prior specific permission and/or a fee. Request permissions from permissions@acm.org.

IWCTS'17, November 7-10, 2017, Redondo Beach, CA, USA

(c) 2017 Association for Computing Machinery.

ACM ISBN 978-1-4503-5491-2/17/11...\$15.00

https://doi.org/10.1145/3151547.3151554

\section{INTRODUCTION}

Recent years have witnessed a tremendous growth in the on-demand economy attracting million of consumers annually and over $\$ 50$ billion in spending [8]. Even more growth is expected with emerging technologies such as self-driving cars, which can have a transformative impact on on-demand services such as ride-sharing and package and delivery services [4, 36], making people's lives much more convenient. This growing economy implies a growing need for designing brokers the can efficiently match the increasing on-demand traffic from consumers to service providers. Table 1 presents a taxonomy of different types of current and envisioned on-demand spatial services. In this context, a spatial service refers to a category of services where the locations of consumers and service providers are critical in the matching process since both parties need to meet for the service to take place. As shown in the table, services are classified based on whether consumers and service providers are stationary or moving. Many widely used on-demand services lie within the top right corner with stationary consumers and moving service providers. This includes ride-hailing services, food delivery services such as Instacart [13] and Doordash [9], bike rental (e.g., Spinlister [3]), home services (e.g., TaskRabbit [4]), and dry-cleaning (e.g. Rinse [27]), etc. Another category of on-demand services are services which involve moving consumers and stationary service providers. For instance, some mobile applications [25, 32] allow users to search for products in nearby stores and locate them down to the aisle. However, matching consumers to their nearest store may lead to long wait times since stores in highly populated regions may exhibit longer lines during periods of high demand (e.g. busy lunch hours, dinner on Friday nights, or Black Friday sales events). On-demand spatial service brokers may help direct mobile consumers to service providers that minimize their waiting times [2]. A third category of on-demand services involves mobile consumers and mobile service providers. This category may include food trucks and delivery vehicles delivering products to consumers in their cars, as well as applications for on-the-go sharing of items and products (e.g. jumper cables). In this paper, we focus on generating synthetic datasets for on-demand services with moving consumers and stationary service providers. We use a restaurant reservation service as an example for evaluating our proposed work. 
Table 1: Taxonomy of On-demand Services

\begin{tabular}{|c|c|c|c|}
\hline & & \multicolumn{2}{|c|}{ Service Providers } \\
\hline & & Stationary & Moving \\
\hline \multirow[t]{2}{*}{ Consumers } & Stationary & & $\begin{array}{l}\text { e.g. ride-hailing services, food delivery, } \\
\text { on-demand dry cleaning, beauty services } \\
\text { (e.g., [34]), and dog-walking (e.g. [39]) }\end{array}$ \\
\hline & Moving & $\begin{array}{l}\text { e.g. shopping cart pickup, salon } \\
\text { reservation, dinner reservation on Friday } \\
\text { nights, on-demand Black Friday shopping }\end{array}$ & e.g. food trucks, on-the-go product sharing \\
\hline
\end{tabular}

Designing robust spatial on-demand service brokers poses several important research questions:

- How to design on-demand matching strategies that are robust against variations in the supply-demand balance? Studying this research question is essential for designing robust on-demand brokers since supply and demand exhibit spatio-temporal heterogeneity. Therefore, a matching strategy that may work optimally for a specific time and/or location may not be optimal for other times/locations with different variations of the supply-demand balance.

- How to ensure balanced service provider utilization to keep the eco-system functioning? An on-demand broker has to keep its "eco-system" alive by engaging many service providers and providing equitable opportunities for providers to fulfill the available demand. For instance, in an on-demand ride-hailing service, drivers that are consistently not assigned any rides may eventually decide to leave the system or switch to another broker, resulting in service degradation and market collapse. The importance of keeping the supply eco-system functioning has been evident through recent efforts by ride-hailing services to encourage drivers to stay on the road with incentives such as tips, accommodating drivers trip preferences, alerting them when they are close to meeting personal earning targets, and paying for wait times [7, 30].

- How to design pricing mechanisms for regulating supply and demand? Aside from increasing supply by using service provider incentives or sharing information about high demand locations, on-demand brokers may also regulate supply and demand imbalance through designing dynamic pricing mechanisms (e.g. surge pricing).

Addressing the above research questions requires the availability of spatio-temporal data from both the supply and demand sides of the business model. However, such datasets are usually proprietary and not available to the public. Furthermore, real-world public datasets that have been used for evaluating on-demand and location-based services [3, 12, 24, 28] are very limited in their spatial coverage (e.g. New York City [22] and Shanghai [31] Taxi Trip Data); only capture the demand side (e.g. crowdsourced GPS trajectory data such as GeoLife [18]); or not representative of real demand for different service types (e.g. online check-ins [33]). Research on traffic generators $[5,6,10,15,17,21,29,35]$ has addressed the problem of generating synthetic spatio-temporal datasets for various applications such as route finding, object tracking, and motion prediction. However, these generators cannot be used for studying the problem of supply-demand imbalance since they focus only on the demand side.

The difficulty of obtaining real-world or otherwise suitable spatio-temporal datasets hinders the design and evaluation of robust and efficient on-demand service brokers. In this work, we propose a supply and demand aware framework for generating synthetic datasets for evaluating on-demand services involving moving consumers and stationary service providers. The framework focuses on supply and demand simultaneously while also generating datasets that resemble real-world data characteristics (e.g. real-world spatial distribution of supply and demand) by leveraging multiple publicly available data sources. The rest of the paper is organized as follows: Section 2 reviews the related work. Section 3 presents our proposed framework for generating synthetic datasets for on-demand services. Section 4 evaluates the quality and performance of the data generator. Finally, Section 5 concludes the paper.

\section{RELATEDWORK}

Research on synthetic spatio-temporal data generation has mainly focused on creating benchmarks for mobile databases, location-based services and applications of traffic monitoring and analysis. These data generators fall into two categories: moving object data generators that assume free movement in Euclidean space, and traffic generators where data is constrained to the road network.

Free Moving Objects Data Generators: An early contribution in this category was the GSTD (i.e. Generate Spatio-Temporal Data) algorithm [35] which generates sets of moving points or rectangles following a user defined input distribution. The algorithm was extended [26] to simulate more complex behaviors such as clustered movements and moving in an obstructed environment. In [37], the generator adds support for complex two-dimensional objects and simulates properties such as the object's color, speed, and the influence of other moving and static objects on its direction and speed. The Oporto generator [29] generates data for simulating fishing ships moving in the direction of fish shoals and avoiding storm areas.

Traffic Generators: This category includes generators of data objects moving along a road network. Brinkhoff [6] proposed a trip-based (i.e. short term observations) generator where the speed of moving objects is affected by external events such as simulated weather changes and traffic jams. BerlinMod [10] 
proposed an object-based (i.e. long-term) simulation where an object is associated with multiple trips over several days. In these generators, the origin and destination nodes of a trip are selected based on a uniform distribution or by relying on user input weights for the different regions. In [5], the generator mimics real-life GPS traces by using statistical profiles derived from historical GPS data. MNTG [21] proposed an extensible web-based traffic generator that acts as a wrapper over existing generators, but allow users to easily configure and use them for arbitrary spatial road regions. In addition, micro-scale simulators [17] have been proposed for simulating vehicle drivers with different driving styles and accounting for parameters such as traffic lights for studying the evolution of traffic in a network. These simulators focus on the accuracy of the proposed models rather than scalability to large data volumes [5].

All the above data generators have only focused on one side of the business model (e.g. demand representing moving consumers). They ignore the supply side, and thus cannot be used for studying on-demand brokers under variations of balanced and unbalanced supply and demand scenarios. In addition, many of the proposed approaches are not representative of real-world demand distribution or rely on historical datasets with limited spatial coverage.

In addition to moving data generators, some studies [14, 16] have focused on generating household and person level population data which can help represent real-world demand distribution. However, these studies still only focus on the demand side. In addition, they rely on population census which only captures the night population, but may not reflect day population affected by daily moving patterns, for instance, due to employment.

\section{PROPOSED FRAMEWORK}

We propose a supply and demand aware framework for generating synthetic datasets for the purpose of designing on-demand spatial service brokers involving moving consumers submitting service requests to stationary service providers. Our framework had two design goals. The first goal is to allow researchers to use the generated spatio-temporal datasets to design on-demand services including designing optimal matching algorithms and algorithms for demand-supply regulation under different variations in the market balance (i.e. supply-demand ratio). The second goal is to ensure that the generated data represents real-world data characteristics by capturing the real-world spatial distribution of supply and demand.

Our proposed framework is illustrated in Figure 1. Researchers can use the framework to generate datasets by specifying the supply-demand ratio to be simulated, which is helpful for market balance scenario planning. In addition, users specify constraints on generating the supply including the range of providers service rates and service type. Users also specify the required arrival time window for the generated consumer requests as well as other required consumer constraints. The framework also leverages several publicly available data sources including road network data for the region under study, census population and employment data for that region, and online business catalogs (e.g. Google
Places API [11]). The framework's two main components are the Supply Generator and the Demand Generator.

\subsection{Supply Generator}

The supply generator module consists of two phases: service providers data extraction and augmentation, and map matching.

Data Extraction and Augmentation: In this phase, data about real-world service providers for the user input service type is collected from online business catalogs. To achieve this goal, we generate search queries to Google Places API, a web Service that allows users to search for places, establishments and points of interest. The search query consists of the service type, and the geographic region. The search results are returned in JSON format and parsed for extracting the service providers' information and their GPS coordinates. An alternative approach is to parse online yellow pages for service provider information in the given region including the provider's address, and then geocode the addresses to retrieve the corresponding latitude and longitude. After data extraction, each service provider record is augmented with a provider's service rate per hour. In our evaluation, service rates were randomly generated as uniformly distributed values over the user's input range.

Map matching: The location of each service provider is then matched to the nearest node in the road network. The output service provider records are then input to the demand generator.

\subsection{Demand Generator}

Demand represents consumer requests that are dynamically submitted to the on-demand service broker. The demand generator has three main phases: a network preprocessing phase, demand size estimation, and consumer requests generation.

Network Preprocessing: In this phase, the road network is preprocessed by associating nodes with their corresponding day and night population values. Night population is directly acquired from publicly available census population datasets, aggregated to the traffic analysis zone (TAZ) level. However, day population needs to be derived by adjusting for variations such as home-to-work trips to allow an accurate spatial distribution of the demand. In our framework, we leveraged employment Origin-Destination (OD) matrices available from the US Census Bureau [38], where each value $c_{i j}$ in the matrix provides a count of workers who move from residence area $i$ to workplace area $j$. The areas in the OD matrix are provided at the census block level. To obtain the night population for a TAZ, the data is first aggregated to the TAZ level. Then, the day population of a TAZ is computed by adding the zone night population and its employment in-flow (i.e. number of workers entering the zone for work) and subtracting the employment out-flow (i.e. number of workers leaving the zone for work). Other higher-resolution population datasets such as the LandScan dataset [23] from Oak Ridge National Laboratory are also available with license fees.

Demand Size Estimation: The generation of demand data (represented by consumer service requests) can be tuned by varying the supply-demand ratio to allow simulating different market conditions. Given a value for the supply-demand ratio $S D R$ as a user input, the average number of consumer requests 


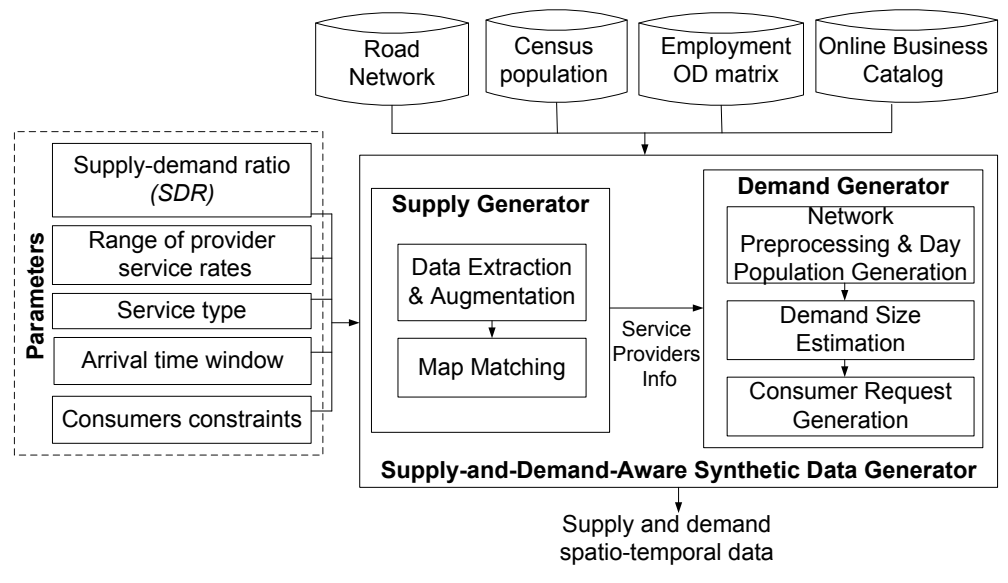

Figure 1: Data Generation Framework

generated per hour $\lambda$ satisfies the following equation:

$$
S D R=\frac{\sum_{p \in P} s_{p}}{\lambda}
$$

where $P$ is the set of all service providers and $s_{p}$ is the service rate per hour of provider $p \in P$.

Consumer Requests Generation Consumer service requests are generated according to the required demand size computed in the previous phase. Each consumer request is associated with an arrival time and the location representing the road network node at which the request originated, in addition to other possible service constraints that may vary according to the type of on-demand service requested (e.g. maximum acceptable wait time). The arrival times of consumer requests are generated within the arrival time window specified by the user, following a Poisson arrival process with intensity equal to $\lambda$ (computed from Equation 1). The generation of the location and other consumer constraints is described as follows:

- Generation of Origin Locations: The number of times each node is selected as an origin location of a consumer request is proportional to the ratio of the node's population to the total population in the study region. For consumer requests with arrival times during the day, the day population of the nodes is used. Similarly, the night population of the nodes is used when selecting the origin locations of requests arriving during night-time.

- Generation of Other Consumer Constraints: Consumer service requests may also have other consumer specified constraints. Examples include a maximum acceptable travel distance, maximum travel time, and/or maximum wait time. Such constraints can also be randomly generated within a user specified range, depending on the type of service being simulated. In addition, since on-demand services require real-time response to users, our generation framework has mainly focused on generating the real-time location at which the request has originated. However, some on-demand applications may also allow the user to specify a destination (e.g. to suggest services along the user's route). One possible approach for generating destination nodes is to employ the spatial distribution of destination nodes obtained from origin-destination matrices.

\section{EVALUATION}

In our earlier work on designing on-demand service brokers [2], we employed the proposed framework for evaluating several matching policies under different market balance variations and showed that the best performing policy is dependent on the relationship between the available supply and demand. In this paper, we evaluate the quality of our proposed framework in terms of its ability to capture real-world spatial demand distribution as indicated by population data. We also evaluated the performance of our generator for different workload sizes. Synthetic datasets were generated for supply and demand representing an on-demand service for lunch and dinner reservation requests in the city of Minneapolis. Figure 2 shows 120 restaurants (i.e. service providers) in Minneapolis, extracted using the Google Places API. Consumer requests were set to arrive within a 10 hour window, including 5 lunch hours and 5 dinner hours. Experiments were performed using road network provided by the Minnesota Department of Transportation [19] and Census population data from the Minnesota GeoSpatial Commons [20]. The employment origin-destination matrix data was provided by the Minnesota Department of Employment and Economic Development [1]. All experiments were run on a machine with an Intel Core i5 $2.3 \mathrm{GHz}$ processor and 8 GB RAM. We used the Java programming language for our implementation.

\subsection{Geographic Validation}

To evaluate how well our demand generation resembled real-life spatial distribution, we compared the spatial distribution of our generated lunch and dinner demand, at different values of the supply-demand ratio $(S D R)$, to the real-world day and night population maps. To simulate different $S D R$ values, we randomly generated a service rate per hour for each restaurant within the range of 10 to 20 requests per hour, resulting in a total of 18,160 service slots. Table 2 shows the details of the datasets generated for the different $S D R$ values. As shown in the table, the generated 


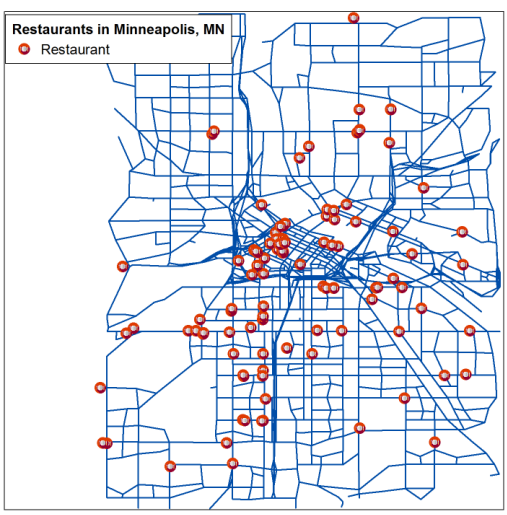

Figure 2: Locations of Restaurants in Minneapolis (Best viewed in color)

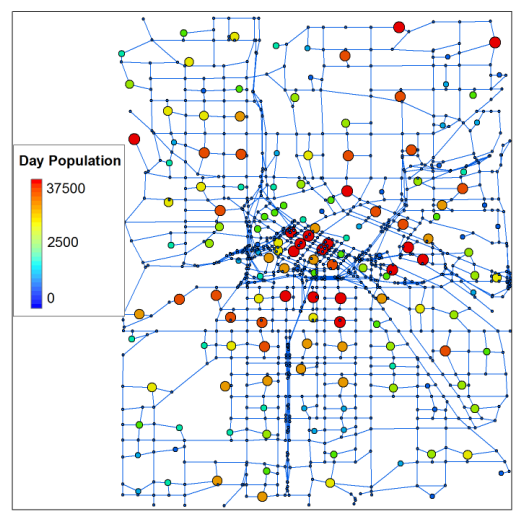

Figure 3: Day Population in Minneapolis (Best viewed in color)

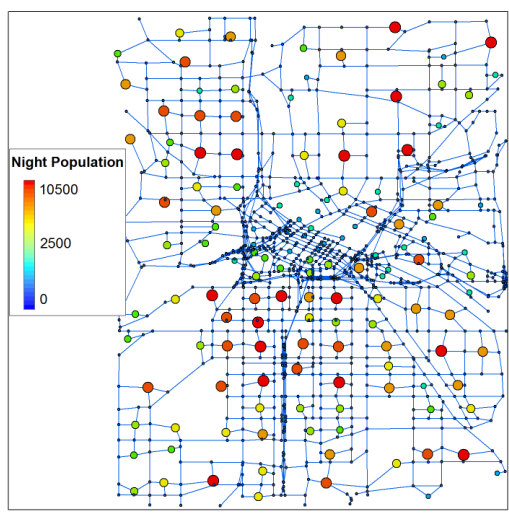

Figure 4: Night Population in Minneapolis (Best viewed in color)

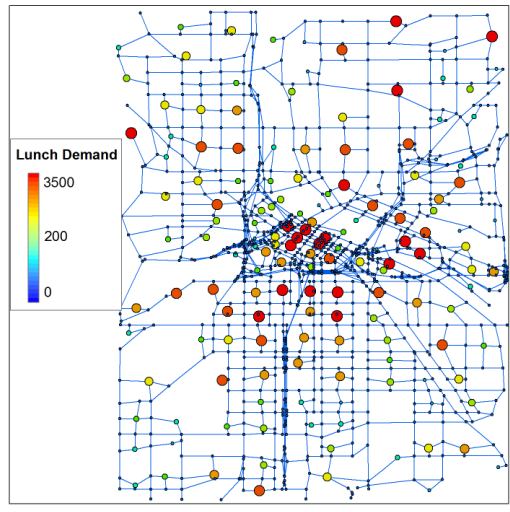

Figure 5: Generated lunch demand for $S D R=0.2$ (Best viewed in color)

Table 2: Details of synthetic dataset used for spatial validation. Total supply is fixed across all $S D R$ values.

\begin{tabular}{|l|l|l|l|}
\hline $\begin{array}{l}\text { Supply-demand } \\
\text { ratio SDR }\end{array}$ & $\begin{array}{l}\text { No. of lunch } \\
\text { requests }\end{array}$ & $\begin{array}{l}\text { No. dinner } \\
\text { requests }\end{array}$ & $\begin{array}{l}\text { Total no. of } \\
\text { requests }\end{array}$ \\
\hline 0.1 & 90,150 & 91,089 & 181,239 \\
\hline 0.2 & 45,149 & 44,997 & 90,146 \\
\hline 1 & 9,038 & 9,135 & 18,173 \\
\hline 5 & 1,769 & 1,789 & 3,558 \\
\hline 10 & 881 & 883 & 1,764 \\
\hline
\end{tabular}

datasets represent different variations of the market balance. For instance, $S D R=0.1$ indicates a scenario where demand greatly exceeds supply, $S D R=1$ indicates a balanced supply and demand scenario, and $S D R=10$ indicates a scenario where the generated supply greatly exceeds the demand.

Figure 3 and Figure 4 show the day and night population in Minneapolis respectively, with larger size nodes indicating a larger population. As observed from the figures, the downtown region (in the figure center) with the central business district has higher population during the day than during the night. Figure 5 and

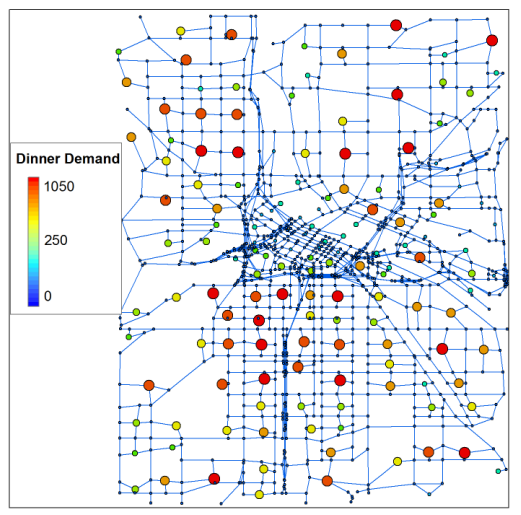

Figure 6: Generated dinner demand for $S D R=0.2$ (Best viewed in color)

Figure 6 show the spatial distribution of our generated lunch and dinner demand (i.e. consumer requests) at each node respectively, for $S D R=0.2$. We can observe that the lunch demand spatial distribution is very similar to the real-world day population, while the dinner demand distribution is similar to the night population. We also generated demand maps for the other SDR values, and they all exhibit similar behavior but were eliminated due to space limitations. The quality of real-world demand representation can be further improved by populating our generic framework with more data sources (e.g. adjusting for school trips).

\subsection{Performance Evaluation}

We evaluated the performance of our proposed framework in terms of the time needed for generating datasets of different sizes (i.e. different SDR scenarios). Figure 7 shows the effect of varying $S D R$ on the execution time of generating demand. In this experiment, we fixed the service rate of each provider $s_{p}$ at 10 , 20 and 30 requests per hour. As $S D R$ increases, the execution time decreases, since a smaller number of consumer requests is generated. At $S D R=0.1$ and $s_{p}=30$ requests/hour, a total of 359,747 
consumer requests were generated in $953 \mathrm{~ms}$ indicating an ability to generate large datasets in reasonable time.

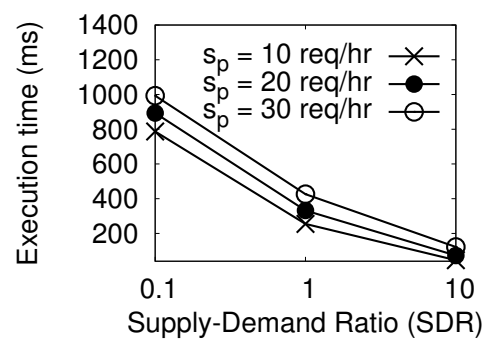

Figure 7: Execution time vs. SDR

\section{CONCLUSION}

In this paper, we proposed a supply and demand aware framework for generating synthetic spatio-temporal datasets that can be used for evaluating the design of on on-demand service brokers involving moving consumers and stationary service providers. Our proposed framework can be used for simulating different variations in the market balance, an essential requirement for designing on-demand brokers. In addition, our framework leverages multiple publicly available datasets that have been missing from other related work studies, allowing our framework to mimic the real-world spatial distribution of supply and demand by considering population data and adjusting for work-to-home and home-to-work trips. Our performance evaluation indicated the ability of our framework to efficiently simulate large demand sizes. In the future, we plan to extend our performance evaluation to larger geographic regions with several service types. In addition, we plan to explore a parallel formulation for the supply and demand generation, possibly by partitioning the study area into geographic subregions.

\section{ACKNOWLEDGMENTS}

This material is based upon work supported by FORD University Research Program (URP), the National Science Foundation under Grant No. IIS-1320580 and 1737633, the USDOE under Grant No. DE-AR0000795, the USDA under Grant No. 2017-51181-27222, and the Minnesota Supercomputing Institute (MSI) at the University of Minnesota (www.msi.umn.edu). We would like to thank Kim Koffolt and the members of the University of Minnesota Spatial Computing Research Group for their comments.

\section{REFERENCES}

[1] Department of Employment and Economic Development. 2017. Data Tools. https://mn.gov/deed/data/data-tools/. (2017).

[2] Reem Y Ali, Emre Eftelioglu, Shashi Shekhar, Shounak Athavale, and Eric Marsman. 2016. Supply-demand ratio and on-demand spatial service brokers: a summary of results. In Proceedings of the 9th ACM SIGSPATIAL International Workshop on Computational Transportation Science. ACM, 7-12.

[3] Jie Bao, Yu Zheng, David Wilkie, and Mohamed Mokbel. 2015 Recommendations in location-based social networks: a survey. GeoInformatica 19, 3 (2015), 525-565.

[4] Joel Barbier. 2017. CNBC. Self-driving cars will disrupt more than the auto industry. Here are the winners and losers. https://www.cnbc.com/2017/05/03/ self-driving-cars- will-disrupt-10-industries-commentary.html. (May 2017).

[5] Konrad Bösche, Thibault Sellam, Holger Pirk, René Beier, Peter Mieth, and Stefan Manegold. 2012. Scalable Generation of Synthetic GPS Traces with Real-Life Data Characteristics.. In TPCTC. Springer, 140-155.
[6] Thomas Brinkhoff. 2002. A framework for generating network-based moving objects. GeoInformatica 6, 2 (2002), 153-180.

[7] Brian X. Chen. 2017. The New York Times. WhatŠs Changing in UberŠs New App. https://www.nytimes.com/2017/06/21/technology/ uber- gratuity-changes-what-you-need-to-know-about.html?mcubz=1. (2017)

[8] Charles Colby and Kelly Bell. 2016. Harvard Business Review. The On-Demand Economy Is Growing, and Not Just for the Young and Wealthy. http://goo.gl/ LyOlHl. (April 2016).

[9] Doordash. 2017. https://www.doordash.com/. (2017)

[10] Christian Düntgen, Thomas Behr, and Ralf Hartmut Güting. 2009. BerlinMOD: a benchmark for moving object databases. The VLDB fournal-The International Journal on Very Large Data Bases 18, 6 (2009), 1335-1368.

[11] Google Places API. 2017. https://developers.google.com/places/. (2017).

[12] Yan Huang et al. 2014. Large scale real-time ridesharing with service guarantee on road networks. Proc. of the VLDB Endowment 7, 14 (2014), 2017-2028.

[13] Instacart. [n. d.]. https://www.instacart.com/. ([n. d.]).

[14] Shubham Jain, Nicole Ronald, and Stephan Winter. 2015. Creating a Synthetic Population: A Comparison of Tools. In Proceedings of 3rd Conference of Transportation Research Group (CTRG) of India, Kolkata, India.

[15] Daniel Krajzewicz, Georg Hertkorn, Christian Rössel, and Peter Wagner. 2002. SUMO (Simulation of Urban MObility)-an open-source traffic simulation. In Proceedings of the 4th middle East Symposium on Simulation and Modelling (MESM20002). 183-187.

[16] Poh Ping Lim and David Gargett. 2013. Population Synthesis for Travel Demand Forecasting. In 36th Australasian Transport Research Forum (ATRF).

[17] Ronghui Liu, Dirck Van Vliet, and D Watling. 1995. DRACULA: dynamic route assignment combining user learning and microsimulation. PTRC-PUBLICATIONS-P (1995), 143-152.

[18] Microsoft.com. 2011. Geolife GPS Trajectory Dataset User Guide. https://goo. gl/zJpFEL. (july 2011).

[19] Minnesota Department of Transportation. 2017. Minnesota MnDOT Interactive GIS Basemap. http://www.dot.state.mn.us/maps/gdma/gis-data.html. (2017).

[20] Minnesota GeoSpatial Commons. 2014. https://gisdata.mn.gov/. (2014).

[21] Mohamed F Mokbel, Louai Alarabi, Jie Bao, Ahmed Eldawy, Amr Magdy, Mohamed Sarwat, Ethan Waytas, and Steven Yackel. 2013. MNTG: an extensible web-based traffic generator. In International Symposium on Spatial and Temporal Databases. Springer, 38-55.

[22] NYC.gov. 2017. TLC Trip Record Data. http://www.nyc.gov/html/tlc/html/ about/trip_record_data.shtml. (september 2017).

[23] Oak Ridge National Laboratory. 2017. LandScan. http://web.ornl.gov/sci/ landscan/. (2017)

[24] Masayo Ota et al. 2015. A scalable approach for data-driven taxi ride-sharing simulation. In IEEE Intl. Conf. on Big Data. IEEE, 888-897.

[25] Sarah Perez. 2014. TechCrunch. Shopping Search Engine TheFind Arrives On Android. https://techcrunch.com/2014/03/06/ shopping-search-engine-thefind-arrives-on-android/. (March 2014).

[26] Dieter Pfoser and Yannis Theodoridis. 2003. Generating semantics-based trajectories of moving objects. Computers, Environment and Urban Systems 27, 3 (2003), 243-263.

[27] Rinse. 2017. https://www.rinse.com/. (2017).

[28] Diego Saez-Trumper, Daniele Quercia, and Jon Crowcroft. 2012. Ads and the city: considering geographic distance goes a long way. In Proceedings of the sixth ACM conference on Recommender systems. ACM, 187-194.

[29] Jean-Marc Saglio and José Moreira. 2001. Oporto: A realistic scenario generator for moving objects. GeoInformatica 5, 1 (2001), 71-93.

[30] NOAM SCHEIBER. 2017. The New York Times. How Uber Uses Psychological Tricks to Push Its DriversŠ Buttons. https://www.nytimes.com/interactive/2017/ 04/02/technology/uber-drivers-psychological-tricks.html?_r=0. (April 2017).

[31] Shanghai Jiao Tang Universitrt, CSE Department. Wireless and Sensor Networks Lab. 2017. Traffic GPS Data. http://wirelesslab.sjtu.edu.cn/taxi_trace_data.html. (2017).

[32] Shops by aisle411. 2017. https://www.aisle411.com/shops/. (2017).

[33] SNAP.Stanford.edu. [n. d.]. Stanford Large Network Dataset Collection: Location-based online social networks. http://snap.stanford.edu/data/\#locnet. ([n. d.]).

[34] StyleBee. 2017. https://www.stylebee.com/. (2017).

[35] Yannis Theodoridis, Jefferson Silva, and Mario Nascimento. 1999. On the generation of spatiotemporal datasets. In Advances in Spatial Databases. Springer, $147-164$.

[36] Transportation Research Board, National Academies of Sciences, Engineering, and Medicine. 2016. Between Public and Private Mobility: Examining the Rise of Technology-enabled Transportation Services. The National Academies Press.

[37] Theodoros Tzouramanis, Michael Vassilakopoulos, and Yannis Manolopoulos. 2002. On the generation of time-evolving regional data. GeoInformatica 6, 3 (2002), 207-231.

[38] United States Census Bureau. 2017. https://www.census.gov/. (2017).

[39] Wag. 2017. https://wagwalking.com/. (2017). 